\title{
Transcriptome profiling reveals the role of ZBTB38 knock- down in human neuroblastoma
}

Jie Chen ${ }^{1,2,3}$, Chaofeng Xing ${ }^{1,2}$, Li Yan ${ }^{4}$, Yabing Wang ${ }^{5}$, Haosen Wang ${ }^{6}$, Zongmeng Zhang ${ }^{1}$, Daolun Yu ${ }^{1}$, Jie Li ${ }^{1}$, Honglin $\mathbf{L i}^{7}$, Jun Li ${ }^{\text {Corresp., }}{ }^{1}$, Yafei Cai ${ }^{\text {Corresp. }}{ }^{2}$

${ }^{1}$ College of Life Sciences, Anhui Provincial Key Lab of the Conservation and Exploitation of Biological Resources, Anhui Normal University, WuHu, China

2 College of Animal Science and Technology, Nanjing Agricultural University, Nanjing, China

3 The Secondary Hospital of Wuhu, WuHu, China

4 Department of Radiation Oncology, Linyi People Hospital, Linyi, China

5 The First Affiliated Hospital of Wannan Medical College, WuHu, China

6 Taizhou 4th Hospital, Taizhou, China

7 Department of Biochemistry and Molecular Biology, Medical College of Georgia, Augusta State University, GA, United States

Corresponding Authors: Jun Li, Yafei Cai

Email address: lijunplant@163.com, ycai@njau.edu.cn

ZBTB38 belongs to the zinc finger protein family and contains the typical BTB domains. As a transcription factor, ZBTB38 is involved in cell regulation, proliferation and apoptosis, whereas, functional deficiency of ZBTB38 induces the human neuroblastoma cell death potentially. To have some insight into the role of ZBTB38 in neuroblastoma development, high throughput RNA sequencing was performed using the human neuroblastoma cell line SH-SY5Y with the deletion of ZBTB38. In the present study, 2,438 differentially expressed genes (DEGs) in ZBTB38-1- SH-SY5Y cells were obtained, $83.5 \%$ of which was downregulated. Functional annotation of the DEGs in the Kyoto Encyclopedia of Genes and Genomes database revealed that most of the identified genes were enriched in the neurotrophin TRK receptor signaling pathway, including PI3K/AKt and MAPK signaling pathway. we also observed that ZBTB38 affects expression of CDK4/6, Cyclin E, MDM2, ATM, ATR, PTEN,Gadd45 and PIGs in the p53 signaling pathway. In addition, ZBTB38 knockdown significantly suppresses the expression of autophagy-related key genes including PIK3C2A and RB1CC1. The present meeting provides evidence to molecular mechanism of ZBTB38 modulating neuroblastoma development and targeted anti-tumor therapies. 
1 Transcriptome Profiling Reveals the Role of ZBTB38

2 Knock-down in Human Neuroblastoma

4 Jie Chen ${ }^{1,2,3}$, Chaofeng Xing ${ }^{1,2}$, Li Yan ${ }^{4}$, Yabing Wang ${ }^{5}$, Haosen Wang ${ }^{6}$, Zongmeng Zhang ${ }^{1}$,

5 Daolun $\mathrm{Yu}^{1}$, Jie $\mathrm{Li}^{1}$, Honglin $\mathrm{Li}^{7}$, Jun $\mathrm{Li}^{1}$, Yafei Cai ${ }^{2}$

6

$7{ }^{1}$ College of Life Sciences, Anhui Provincial Key Lab of the Conservation and Exploitation of

8 Biological Resources, Anhui Normal University, Wuhu, China

$9{ }^{2}$ College of Animal Science and Technology, Nanjing Agricultural University, Nanjing, China

$10{ }^{3}$ The Secondary Hospital of Wuhu, Wuhu, China

$11{ }^{4}$ Department of Radiation Oncology, Linyi People Hospital, Linyi, China

125 The First Affiliated Hospital of Wannan Medical College, Wuhu, China

$13{ }^{6}$ Taizhou 4th Hospital, Taizhou, China

$14{ }^{7}$ Department of Biochemistry and Molecular Biology, Medical College of Georgia, Augusta

15 State University, GA, United States

\section{ABSTRACT}

ZBTB38 belongs to the zinc finger protein family and contains the typical BTB domains. As a transcription factor, ZBTB38 is involved in cell regulation, proliferation and apoptosis, whereas, functional deficiency of ZBTB38 induces the human neuroblastoma cell death potentially. To have some insight into the role of ZBTB38 in neuroblastoma development, high throughput RNA sequencing was performed using the human neuroblastoma cell line SH-SY5Y with the deletion of ZBTB38. In the present study, 2,438 differentially expressed genes (DEGs) in ZBTB38 ${ }^{-/-}$SH-SY5Y cells were obtained, $83.5 \%$ of which was down-regulated. Functional annotation of the DEGs in the Kyoto Encyclopedia of Genes and Genomes database revealed that most of the identified genes were enriched in the neurotrophin TRK receptor signaling 
27 pathway, including PI3K/Akt and MAPK signaling pathway. we also observed that ZBTB38 28 affects expression of CDK4/6, Cyclin E, MDM2, ATM, ATR, PTEN,Gadd45 and PIGs in the 29 p53 signaling pathway. In addition, ZBTB38 knockdown significantly suppresses the expression 30 of autophagy-related key genes including PIK3C2A and $\mathrm{RB} 1 \mathrm{CC} 1$. The present meeting provides 31 evidence to molecular mechanism of ZBTB38 modulating neuroblastoma development and 32 targeted anti-tumor therapies.

33

Neuroblastoma (NB) is an embryonal malignant tumor that originates from the neural crest cells of the sympathetic nervous system. NB is the most common extracranial solid tumor in children, accounting for $8 \%-10 \%$ of pediatric malignancies (Castel et al. 2013; Schulte et al. 2013). This disease is highly malignant and progresses rapidly — most of the patients would have been at the advanced stage upon diagnosis when conventional radiotherapy and chemotherapy would feature low efficacy, thus resulting in extremely low survival rate (Bagatell \& Cohn 2016). In recent years, new therapeutic methods, including haematopoietic stem cell transplantation and biological immunotherapy, have been employed to treat relapsed or refractory NB, but their efficacy remains limited (Binkhathlan \& Lavasanifar 2013; Han \& Wang 2015). Drug resistance has been recognised as the key obstacle to reaching a satisfactory outcomes (Castel et al. 2013), whereas the induction of programmed death of the cancer celldeath by targeted gene therapy shows notable potential in improving the cure rate and long-term survival of NB patients, especially those with higher risks.

ZBTB38 belongs to the zinc finger and BTB domain-containing protein family. Most members of the family, as transcription factors, bind to specific DNA sequences and regulate the transcriptional activity of target genes (Sasai et al. 2005; Stogios et al. 2005). The ZBTB family members also participate in various intracellular signal transduction pathways via recognising and interacting with other proteins, thereby playing important roles in the transcriptional repression, DNA damage, tumorigenesis, cell proliferation, differentiation and apoptosis (Lee et al. 2010; Matsuda et al. 2008; Nishii et al. 2012). At least 49 ZBTB proteins are encoded in the human genome; most of them are nuclear proteins (Lee \& Maeda 2012). Among the predicted BTB domain-containing proteins encoded by the human genome, only several of them have been functionally characterised (Cai et al. 2012; Matsuda et al. 2008). No relevant studies have been reported concerning the effect of ZBTB38 on human NB.

Transcriptomic studies have progressed rapidly over the recent years, which, contrary to the research on an individual genes, enabled the investigation on the altered expression of 
60 differentially expressed genes (DEGs) at the level of whole protein-coding or non-coding RNAs

61 in cells or tissues of the body. Transcriptomic studies can also provide information on the

62 relationship between the transcriptional regulation and the protein functions in the whole genome

63 under specific conditions (Reimann et al. 2014; Zhao et al. 2011). Next-generation sequencing

64 (NGS) technology offers important technical support for the annotation and quantification of

65 transcriptomes. The major strength of this technique lies in its high throughput and high

66 sensitivity for transcript abundance, thus providing thorough understanding of the transcriptional

67 genome information in a comprehensive manner and valuable resources for the investigation of

68 the therapeutic biomarkers of cancer (Chang et al. 2015; Li et al. 2014).

69 Therefore, in the present study, a high-throughput transcriptome sequencing approach was

70 adopted to investigate the transcriptome profiles of NB cells in which the expression of ZBTB38

71 gene was down-regulated, thus revealing the potential biomarkers associated with anti-tumor

72 therapies for NB.

\section{MATERIALS \& METHODS}

\section{Cell culture and standard assays}

SH-SY5Y cells were purchased from American Type Culture Collection (Rockville, MD, USA) and cultured in Dulbecco's modified Eagle's medium supplemented with 10\% fetal bovine serum and penicillin-streptomycin. siRNAs were used to deplete ZBTB38 gene in SH-SY5Y cells by lipofection. Scramble siRNA ( siNC) were used as negative control for knockdown experiment. Transient transfections, quantitative real-time polymerase chain reaction (qRT-PCR) and western blotting were performed as described previously (Cai et al. 2012; Cai et al. 2017). GAPDH and $\beta$-actin (for human samples) were used as the internal control. The primers used in qRT-PCR and siRNA suppression assays are listed in supplemental Table S1.

Cell viability was tested using the CCK-8 assay. The absorbance of each well was measured at $450 \mathrm{~nm}$ on a microplate reader. The proliferation rate was defined in terms of the percentage of each group of surviving cells compared with the untreated group for both cell lines. measuring mono and oligonucleosomes in the lysates of apoptotic cells according to the manufacturer's protocol. 
90 RAPTOR, LC3B, p62 and RB1CC1 antibodies were procured from Abcam plc (Massachusetts, 91 US). In Situ Cell Death Detection Kit-POD was purchased from Roche (Basel, Switzerland).

92

93

94

95

96

97

98

99

100

101

102

103

104

105

106

107

108

109

110

111

112

113

114

115

116

117

118

119

120

121

122

\section{RNA Preparation and library construction for transcriptome sequencing}

Transcriptome high-throughput sequencing was performed in the control group (SH-SY5Y cells transfected with liposome alone, Samples-ID: T04, T05, T06) and the treatment group (SHSY5Y cells transfected with ZBTB38 siRNA, Samples-ID: T01, T02, T07). Total RNA was isolated from SH-SY5Y cells using TRIzol and the pure-link RNA mini kit (ThermoFisher Scientific, Waltham, MA, USA) according to manufacturer's instructions. RNA purity was checked using the NanoPhotometer spectrophotometer (IMPLEN, CA, USA). RNA concentration was measured using the Qubit RNA Assay Kit in Qubit 2.0 Fluorometer (Life Technologies, CA, USA). RNA integrity was assessed using the RNA Nano 6000 Assay Kit of the Agilent Bioanalyzer 2100 system (Agilent Technologies, CA, USA).

In total, $2 \mu \mathrm{g}$ RNA per sample was used as input material for RNA sample preparations. This study included two groups of three biological replicates. Sequencing libraries were generated using a NEBNext UltraTM RNA Library Prep Kit for Illumina (NEB, USA), and index codes were added to attribute sequences to each sample. Fragmentation was performed using divalent cations under elevated temperature in NEBNext First Strand Synthesis Reaction Buffer $(5 \times)$. First-strand cDNA was synthesized using random hexamer primer and M-MuLV Reverse Transcriptase (RNase H). Second-strand cDNA synthesis was subsequently performed using DNA Polymerase I and RNase H. Remaining overhangs were converted into blunt ends via exonuclease/polymerase activities. After the adenylation of $3^{\prime}$ ends of DNA fragments, NEBNext Adaptor with a hairpin loop structure was ligated to prepare for hybridization. The library fragments were purified using AMPure XP system (Beckman Coulter, Beverly, USA). Then, 3 $\mu l$ USER Enzyme (NEB, USA) was used with size-selected, adaptor-ligated cDNA at $37^{\circ} \mathrm{C}$ for $15 \mathrm{~min}$, followed by $5 \mathrm{~min}$ at $95^{\circ} \mathrm{C}$ before PCR. Following this, PCR was performed with Phusion High-Fidelity DNA polymerase, universal PCR primers, and index (X) Primer. Finally, PCR products were purified (AMPure XP system), and library quality was assessed using the Agilent Bioanalyzer 2100 system. The clustering of the index-coded samples was performed on a cBot Cluster Generation System using the TruSeq PE Cluster Kit v4-cBot-HS (Illumia). Following cluster generation, the library preparations were sequenced on an Illumina Hiseq 2500 platform, and paired-end reads were generated.

\section{Data and Statistical Analysis}

\section{Cancer genomics analysis}


123 we downloaded the mRNA expression data from the cancer genome atlas (TCGA)database, and

124 systematically evaluated the expression of ZBTB38 and correlation with patients' survival in 125 tumors of the TCGA database.

126

127

128

129

130

131

132

133

134

135

136

137

138

139

140

141

142

143

144

145

146

147

148

149

150

151

152

153

Quality control

Raw reads of fastq format were firstly processed through in-house perl scripts. In this step, clean reads were obtained by removing reads containing adapter, reads containing ploy-N and low quality reads from raw reads. At the same time, Q20, Q30, GC-content and sequence duplication level of the clean reads were calculated. All the downstream analyses were based on clean reads with high quality (Ewing \& Green 1998; Ewing et al. 1998). The clean data of this article are publicly available in the NCBI sequence reads archive (SRA) with accession number SRP150042.

\section{Comparative analysis}

The adaptor sequences and low-quality sequence reads were removed from the data sets. Raw sequences were transformed into clean reads after data processing. These clean reads were then mapped to the reference genome sequence. Only reads with a perfect match or one mismatch were further analyzed and annotated based on the reference genome. Tophat 2 tools soft were used to map with reference genome (Kim et al. 2013; Langmead et al. 2009). Reference genome download address:ftp://ftp.ensembl.org/pub/release-80/fasta/homo sapiens/.

\section{Gene functional annotation}

The assembled sequences were compared against the NR (NCBI non-redundant protein $\underline{\text { sequences}), ~ P f a m ~(P r o t e i n ~ f a m i l y), ~ K O G / C O G ~(C l u s t e r s ~ o f ~ O r t h o l o g o u s ~ G r o u p s ~ o f ~ p r o t e i n s), ~}$ Swiss-Prot (A manually annotated and reviewed protein sequence database), KO (KEGG Ortholog database), and GO (Gene Ontology) databases with an E-value $\leq 10^{-5}$ for the functional annotation. The Blast2GO program was used to obtain $\mathrm{GO}$ annotation of unigenes including molecular function, biological process, and cellular component categories (Gotz et al. 2008).

\section{Differential expression analysis}

Differential expression analysis of the two conditions was performed using the DEGseq R package (Robinson et al. 2010). The P-values obtained from a negative binomial model of gene expression were adjusted using Benjamini and Hochberg corrections to control for false discovery rates (Anders \& Huber 2010). Genes with an adjusted P-value $<0.05$ were considered to be differently expressed between groups. DEG expression levels were estimated by fragments 
154 per kilobase of transcript per million fragments mapped (Florea et al. 2013). The formula is 155 shown as follow:

156

$$
\mathrm{FPKM}=\frac{\mathrm{cDNA} \text { Fragments }}{\text { Mapped Fragments(Millions) } \times \text { Transcript Length }(\mathrm{kb})}
$$

157

158

159

160

161

162

163

164

165

166

167

168

169

170

171

172

173

174

175

176

177

178

179

180

GO enrichment and KEGG pathway enrichment analysis

GO enrichment analysis of the differently expressed genes (DEGs) was implemented in the "GOseq" package in R based on a Wallenius non-central hyper-geometric distribution, which can adjust for gene length bias in DEGs (Young et al. 2010).

KEGG is a database for understanding high-level functions and utilities of biological systems through large-scale molecular datasets generated by genome sequencing and other highthroughput experimental technologies (http://www.genome.jp/kegg/) (Kanehisa et al. 2008). We used the KOBAS software to test for the statistical enrichment of differentially expressed genes in KEGG pathways. KEGG enrichment can identify the principal metabolic pathways and signal transduction pathways of DEGs (Mao et al. 2005).

\section{DEGs quantitative real-time pcr ( $q R T-P C R)$ verification}

For validation of the transcriptome result, we subjected three significantly differential expressed unigenes on related pathways to qRT-PCR analysis. Redundant RNA from the cDNA library preparation was used to perform reverse transcription according to the Invitrogen protocol. quantitative real-time polymerase chain reaction (qRT-PCR) were performed as described previously (Zhang et al. 2017). The primers used in qRT-PCR suppression assays are listed in Table S1.

\section{Statistical analysis}

All data were reported as mean \pm standard deviation and analyzed using one-way analysis of variance in SPSS v.17.0. Statistical tests were performed with the Kruskal-Wallis and MannWhitney U-tests. A least significant difference test was used for comparisons between groups. A $\mathrm{P}$-value $<0.05$ was considered statistically significant.

\section{RESULTS}

\section{Variation of ZBTB38 in tumors}


181 According to the statistical analysis of the TCGA database resources, we found that the

182 expression changes of ZBTB38 gene are closely related to the occurrence of 20 kinds of cancers,

183 and especially the most remarkable down-regulated expression in uterine corpus endometrial

184 carcinoma (UCEC) and cervical squamous cell carcinoma and endocervical adenocarcinoma

185 (CESC) $(p<0.05)$ (Fig. 1). However, in the prognosis of these 20 tumors, we only uncover here

186 that low expression of ZBTB38 was associated with improved the prognosis of the brain lower

187 grade glioma (LGG) patients (Fig. 2), suggesting that these changes are closely related to

188 neuronal tumors.

189 Neuroblastoma cell proliferation and viability after down-regulated of ZBTB38 expression

190 To investigate the importance of ZBTB38 in theprocess of neuronal tumors, three pairs of 191 siRNAs named siRNA1, siRNA2, and siRNA3, were designed to suppress expression of 192 ZBTB38 in human neuroblastoma cells SH-SY5Y. The protein level of ZBTB38 was decreased 193 significantly $(p<0.05)$ at $24 \mathrm{~h}$ after transfection (Fig. $3 A$ and $B)$, and furthermore, siRNA3 194 worked best for the suppression. No significant difference in cell proliferation and viability was 195 observed among the initial phases of each group after culture by transient transfection $(p>0.05)$. 196 From 12-72 h, the ZBTB38 ${ }^{-/-}$SH-SY5Y group showed significantly lower cell proliferation and 197 viability than the control group $(p<0.05)$ (Fig. 3C and D). Whereas, a sharp increase in 198 apoptosis of SH-SY5Y cells were observed following ZBTB38 siRNA exposure (Fig. 4A).We 199 next determined the expression levels of pro-apoptotic genes in ZBTB38 knockdown cells 200 compared with knockdown control cells. The knockdown of the ZBTB38 gene resulted in a 201 decrease in the expression levels of Noxa, Bak, Bim, Puma and DR5 genes, with significant 202 differences in Noxa, Bim, and DR5 ( $p<0.05)$ (Fig. 4B). These data indicate that inhibition of 203 ZBTB38 triggers apoptosis of NB cells.

\section{Quality control and yield statistics of transcriptome sequencing data}

205 A total of $47.05 \mathrm{~Gb}$ clean data were obtained through the transcriptome sequencing of SH-SY5Y 206 cells, with at least $6.12 \mathrm{~Gb}$ and a $\geq 89.30 \%$ Q30 percentage for each sample (Table 1). Efficiency 207 of sequence alignment referred to the percentage of mapped reads in the clean reads, which 208 reflected the utilization of transcriptome sequencing data. Statistical analysis of the alignment 209 results showed that the efficiency of read alignment for the reads of each sample and the 210 reference genome ranged between $79.42 \%$ and $81.92 \%$ (Table 1), which guaranteed that the 211 selected reference genome assembly was qualified for data analysis.

212 Qualified transcriptome libraries are a major requisite for transcriptome sequencing. To ensure 213 the quality of the libraries, quality of the transcriptome sequencing libraries was evaluated from 214 three different perspectives: 
215 (1) Randomicity of mRNA fragmentation and the degradation of mRNA were evaluated by 216 examining the distribution of inserted fragments in genes. As shown in Figure S1, The

217 degradation of mRNAs was relatively low in the 6 groups of samples.

218 (2) The dispersion degree of the inserted fragment length directly reflected the efficiency of 219 magnetic bead purification during library preparation. Simulated distribution of the inserted 220 fragment length for each sample showed only single-peak pattern, indicating a high purification 221 rate (Fig. S2).

222 (3) With the increase of sequencing data, the number of DEGs tended to saturate, as shown in 223 Figure S3, which confirmed that the data were sufficient and qualified for the subsequent 224 analysis.

\section{DEG and DEGs Function annotation}

226 To acquire the comprehensive genetic information of ZBTB38 ${ }^{--}$SH-SY5Y cells, the unigenes 227 were blasted against the NR, Swiss-Prot, GO, COG, KOG, Pfam, KEGG database resources to 228 identity the functions of all of the unigene sequences. All of DEGs were annotated to genes 229 having known functions in the indicated databases based on the sequences with the greatest 230 similarity. DEseq was used to analyze the DEGs derived from the two groups of cells to obtain a 231 DEGs set. Finally, a total of 2,036 (83.5\%) down-regulated DEGs and 402 (16.5\%) up-regulated 232 DEGs were selected (Table S2 and Fig.S4). The number of DEGs annotated in this gene set was 233 shown in Table 2.

234 A total of 2,258 (93.4\%) DEGs were annotated successfully by GO annotation. These annotated 235 DEGs were classified into the next terms of three ontologies: BP (biological process), CC 236 (cellular component) and MF (molecular function). The distribution of unigenes is shown in 237 Figure 5. Among the "Biological Process", a high percentage of genes were classified into 238 Cellular Process (1,924 unigenes, 85.2\%). Within the cellular component category, the majority 239 of genes were assigned into Cell Part (2,145 unigenes, 95\%). For the molecular function, most 240 of genes were involved in "Binding" (1,949 unigenes, 86.3\%). The greatest number of annotated 241 unigenes were involved in Biological Process. The results of the topGO functional enrichment 242 analyses of DEGs indicated that the most significantly enriched GO terms focus on 243 "neurotrophin TRK receptor signaling pathway" (Table 3).

244 The unigenes was blasted against the COG database in order to orthologously classify gene 245 products. COG classification statistical results of DEGs were shown in Figure 6. In addition to 246 "General function prediction only", "Replication, recombination and repair" accounted for the 247 largest proportion of unigenes (180 DEGs, 13.06\%), followed by "Transcription" (133 DEGs, 248 9.65\%), "Signal transduction mechanisms"(128 DEGs, 9.29\%), "Translation, ribosomal structure 249 and biogenesis" (80 DEGs, 5.81\%), "Posttranslational Modification, Protein Turnover and 
250 Chaperones" (79 DEGs, 5.73\%), "cell cycle control, cell division, and chromosome partitioning" 251 (44 DEGs, 2.98\%). According to the annotation results of the DEGs KEGG database, the largest 252 proportion of the unigenes were involved in the "MAPK signaling pathway" and "PI3K-Akt 253 signaling pathway" of "Environmental Information Processing”(Fig. 7).

254 Based on the results above, a large number of DEGs were screened after a comparative analysis 255 of relevant databases. Meanwhile, functional annotation was also carried out that was crucial for 256 the further understanding of the cellular functions of ZBTB38 gene as a transcription factor.

257 Detection of candidate genes and analysis of the results of Real-time quantitative PCR

258 We analyzed whether the differentially expressed genes were over-presented on a pathway by 259 enrichment of DEGs KEGG pathway (Fig.S5), taking FPKM as a measure for the level of 260 transcripts or gene expressions, DEGs in the p53 signaling pathway, including CDK4/6 261 (ENSG00000105810), Cyclin E (ENSG00000175305), MDM2 (ENSG00000135679), ATM 262 (ENSG00000149311), ATR (ENSG00000175054), PTEN (ENSG00000171862), were down263 regulated, and Gadd45 (ENSG00000179271) and PIGs (ENSG00000115129) were up-regulated 264 (Table S2 and Fig.S5).

265 Top 20 down-regulated unigenes associated with autophagy were selected (Table S3), among 266 which PIK3C2A was the most down-regulated one, followed by RB1CC1 gene. In summary, the 267 transcription factor ZBTB38 is involved in the process of protein synthesis and also, as a positive 268 regulatory factor, in the occurrence of autophagy directly.

269 To validate the sequencing results obtained by RNA-seq, real-time quantitative PCR was 270 performed on three candidate genes, including PIK3C2A, RB1CC1, ATM, related to the mTOR 271 signaling pathway. The result showed that the expression of these candidate genes was 272 significantly decreased in the ZBTB38 ${ }^{-/-}$cells compared to control group, which was similar to 273 the RNA-seq data (Fig. 8). The result verified the reliability of the transcription sequencing 274 results.

275 To further explore the mechanism involved in these events, we examined the expression levels of 276 autophagy genes, in the presence or absence of ZBTB38. The expression levels of LC3B and 277 RB1CC1 were significantly decreased in human NB cells after ZBTB38 knockdown, compared 278 with those from empty liposome-treated SH-SY5Y cells (Fig. 9), which indicate that autophagy 279 is inhibited. In addition, we also detected PTEN and RAPTOR, which are key genes in mTORC1 280 regulation of autophagy signaling pathway, their expression levels were decreased in ZBTB38 281 siRNA-treated SH-SY5Y cells, whereas p62 expression was increased. These results indicate 282 that ZBTB38 loss-mediated autophagy inhibition is likely associated with activation of the 283 mTORC1 signaling pathway. In summary, the transcription factor ZBTB38 is involved in the 284 process of protein synthesis and also, as a positive regulatory factor, in the occurrence of 285 autophagy directly. 


\section{DISCUSSION}

287 Transcriptomic studies have progressed rapidly in recent years. Based on the information of the 288 whole mRNAs obtained in one cell or tissue, the transcriptomic studies provide data on the

289

290

291

292

293

294

295

296

297

298

299

300

301

302

303

304

305

306

307

308

309

310

311

312

313

314

315

316

317

318

319

expression regulation systems and protein functions of all genes. NGS facilitates the deep sequencing of whole cancer genomes for the discovery of novel therapeutic biomarkers, helping to consequently build a solid foundation for the comprehensive studies of cancer

pharmacogenetics. Furthermore, NGS allows for detailed analyses of the whole epigenome and transcriptome, thus profoundly revealing the multilevel regulation networks of the human genome (McGettigan 2013; Wang et al. 2009; Young et al. 2010). Remarkably, the large amount of data on gene expression profiles revealed by transcriptome sequences have provided valuable resources for studies investigating the therapeutic biomarkers of cancer.

The genomic instability is an important factor in the early stages of cell carcinogenesis. This property primarily results from the error-prone DNA repair and the accumulation of abnormal DNA after repair are the main causes of genomic instability that follows (Lord \& Ashworth 2012). The strict regulation of gene transcription is an essential factor to maintain the genomic stability (Kakarougkas et al. 2014). According to the statistical analysis of the TCGA database, the ZBTB protein family is mainly involved in the expression regulation of the target genes. The amplification, deficiency and/or mutation of most genes in the ZBTB family occurs in different types of tumors (Jardin et al. 2007; Maeda et al. 2007; Phan \& Dalla-Favera 2004). Among ZBTB genes, the expression changes of ZBTB38 gene are closely related to the occurrence of 20 kinds of cancers (Fig. 1A), and different tumors exhibit significant differential expression changes, especially the remarkably down-regulated expressions of UCEC and CESC (Fig. 1B and $1 D$ ). However, in our study, the statistical analysis of the prognosis of the LGG patients exhibited a negative correlation with the expression changes of ZBTB38 (Fig. 2), indicating a significant concern regarding the study of the effects of ZBTB38 expression changes on the occurrence and development of neuroma. However, there was no relevant report focused on the expression change of ZBTB38 in NBs. This study demonstrated for the first time that the in vitro knockdown of ZBTB38 seriously affected the proliferation of NB cells. Accordingly, the biological function of ZBTB38 and its relationship with the clinical prognosis of NB deserves further analysis.

The annotation of the DEGs function revealed that after ZBTB38 knockdown, the most of DEGs were enriched in the neurotrophin TRK receptor signaling pathway. Neurotrophic factors (NTs) are a class of factors that regulate neuronal development, differentiation and function. NTs may activate two types of receptors, the high-affinity tyrosine kinase family TRK receptors and the 
320 low-affinity p75 neurotrophin receptor (p75NTR) of the tumor necrosis factor receptor

321 superfamily (Yang et al. 2016). NTs can initiate various complex signal transduction pathways

322 by activating these types of receptors and thus exert biological effects. In most cases, p75NTR is

323 a ligand-activated apoptotic receptor, which primarily induces neuronal apoptosis and activate

324 the apoptotic JKN-p53-Bax signal transduction pathway (Redden et al. 2014). TRKs mainly

325 activate two pathways: the phosphoinositide 3-kinase (PI3K)-Akt signaling which inhibits the

326 production and activity of apoptotic proteins, and the mitogen-activated protein kinase (MAPK)

327 signaling pathway, which activates the anti-apoptotic proteins to promote survival (Wong et al.

328 1999). In this study, the KEGG pathway enrichment analysis of differentially expressed genes

329 revealed that DEGs were the most enriched genes in the MAPK and PI3K-Akt signaling

330 pathways and mostly down-regulated. Thus, we speculated that ZBTB38 knockdown-induced

331 reduction in viability and proliferation rate of SH-SY5Y cells may be closely related to these

332 pathways. We plan to focus on the key components of DEGs in future studies to clarify the

333 related molecular mechanism and to further evaluate the potential of ZBTB38 as a target gene to

334 treating NB.

335 A key feature of NB is that it is a uniform p53 wild-type at diagnosis with intact intrinsic and

336 extrinsic apoptotic mechanisms; direct inactivation of p53 mutations, which are rare, regardless

337 of the stage of treatment, suggests that NBs feature an innate requirement for a baseline p53

338 activity (Kim \& Shohet 2009). In the present study, the KEGG pathway enrichment analysis of

339 DEGs revealed that in the p53 signaling pathway, genes, including CDK4/6, Cyclin E, MDM2,

340 ATM, ATR, PTEN, were down-regulated, and Gadd45 and PIGs were up-regulated after the

341 knockdown of ZBTB38 (Fig. 10). Both the CDK4/6-Cyclin D and the CDK2-Cyclin E

342 complexes serve as the central links in cell cycle regulation via regulating the G1-S transitions

343 in cells, and abnormal activation of the CyclinD-CDK4/6-INK4-Rb pathway, which is often

344 observed in various malignancies, will lead to uncontrolled growth of cancer cells (Sawai et al.

345 2012; The et al. 2015; VanArsdale et al. 2015). In addition, members of the Gadd45 family serve

346 as key regulatory genes in DNA damage repair pathway with p53 as the central link, whereas the

347 upregulation of Gadd45 plays an important role in the regulation of G2/M cell cycle checkpoints

348 and the maintenance of genomic stability to inhibit the cell transformation and the malignant

349 tumor progression (Wang et al. 1999). ATM and ATR belong to the inositol trisphosphate kinase

350 family, both of which can be activated by DNA damage to phosphorylate the downstream

351 substrates such as CHK1, CHK2, and p53. In addition, the down-regulation of both kinases may

352 impair the downstream transmission of the molecular signals and inhibit the p53 activity

353 (Abraham 2001; Matsuoka et al. 2007). MDM2 regulates the function of p53 via two

354 approaches, i.e., mediating the p53 degradation and inhibiting its transcriptional activity. As a

355 negative feedback regulator of $\mathrm{p53}$, the inhibited expression of MDM2 can enhance the 
356

357

358

359

360

361

362

363

364

365

366

367

368

369

370

371

372

373

374

375

376

377

378

379

380

381

382

383

384

385

386

387

388

389

390

transcriptional activity of p53 and inhibit tumorigenesis (Shangary \& Wang 2009). PIGs act as the target downstream genes of $\mathrm{p} 53$ for apoptosis regulation, which is critical for cell apoptosis by participating in the synthesis of reactive oxygen species (ROS) and the regulation of oxidative stress (Jin et al. 2017; Lee et al. 2010). We can speculate that when ZBTB38 gene was knocked down, more PIGs are transferred into the nucleus, where cell damage is repaired. Therefore, cellular response to DNA damage increased, and p53 induced ROS production, ultimately promoting the apoptosis of tumor cells. PTEN is a tumor suppressor gene with phosphatase activity. This gene is also an upstream regulatory inhibitor of the PI3K-Akt signal transduction pathway. PTEN is often referred to as a 'switch' molecule in the PI3K-Akt pathway due to its capability, depending on its lipid phosphatase activity, to remove the phosphate group and participate in the regulation of cell activity. Once the expression of PTEN protein is reduced, the dephosphorylation of phosphatidylinositol $(3,4,5)$-trisphosphate (PIP3) decreases. Excessive PIP3 is subsequently accumulates in the cells, and the PI3K/Akt signaling pathway is continuously activated, eventually leading to cell proliferation or uncontrolled apoptosis and finally the occurrence of various diseases (Bleau et al. 2009; Carnero et al. 2008). In summary, studies investigating the roles of ZBTB38 and p53 pathways in growth and apoptosis of NB cells and those involving the intervention of specific signaling pathways may allow us to further understand the mechanisms of NB occurrence and progression, and thus better evaluate and control this paediatric malignancy.

Among all the KEGG pathway enrichment categories, the majority of the DEGs were enriched in the PI3K-Akt signaling pathway, especially the down-regulated ones, with the most significance noted in PIK3C2A and RB1CC1. PIK3C2A is a member of the PI3K family and one of the key molecules in the signal transduction pathway of growth factors. The overexpression of PIK3C2A in cells has been reported to induce the accumulation and assembly of clathrin, which mediates the transport of proteins between cell membranes and the network structure of the Golgi body via regulating the movement of microtubules (Dragoi \& Agaisse 2015; Shi et al. 2016). RB1CC1 (also known as FIP200), with a molecular weight of $200 \mathrm{kD}$, is an interacting protein of the focal adhesion kinase family. As documented in prior studies, autophagy induction is abolished in RB1CC1-deficient cells. RB1CC1 is an important regulatory protein that can acts on the autophagic initiation complex along with the Unc-51 like autophagy activating kinase simultaneously. RB1CC1 is also a key autophagy initiation factor in the mTORC1-dependent signaling pathway (Ganley et al. 2009; Wang et al. 2011; Wei et al. 2009). We also observed downregulation of PTEN, RAPTOR and LC3B expression (Fig. 9). RAPTOR is a specific component of mTORC1, which is negatively correlated with the activation of mTORC1. The downregulation of RAPTOR expression may also indicate the activation of mTORC1 pathway 
391 (Saxton \& Sabatini 2017). Therefore, as revealed in our study, we believe that the loss of ZBTB38 392 gene in SH-SY5Y cells lead to mTORC1-mediated autophagy inhibition.

393 Orthologous assignments of gene products were carried out using the COG database.

394 Corresponding statistical analysis of the results also indicated that the silencing of the ZBTB38

395 gene affected the homeostasis of the whole cell.As a transcriptional factor, ZBTB38 regulates the 396 transcription of intracellular proteins and influenced the expression and transport of proteins in

397 the downstream signaling pathways. The GO functional enrichment analysessuggested that most 398 of the DEGs were involved in "Binding" and "Catalytic Activity" of the molecular function 399 between ZBTB38-/ cells and the controls. This finding also partially explains the biological 400 functions of the key candidate genes enriched in the KEGG pathway, i.e., all of them are specific 401 binding DNAs or proteins that regulate, the transcriptional activity of target genes and are 402 involved in various intracellular signaling pathways.

403 CONCLUSIONS

404 The functional knockdown of transcription factor ZBTB38 effectively inhibited the proliferation 405 and differentiation of NB cells, which may be largely attributed to the significant inhibition of 406 the neurotrophin TRK receptor signaling pathway. In addition, the downregulation of ZBTB38 407 may also promote apoptosis of the NB cells by regulating key components of the p53 signaling 408 pathway. Two DEGs (PIK3C2A and RB1CC1) that closely related to autophagy initiation were 409 significantly inhibited, suggesting that ZBTB38 downregulation also blocked autophagy, an 410 important mechanism that protects the cells from programmed cell death, thus accelerating 411 apoptosis of tumor cells.

\section{ACKNOWLEDGEMENTS}

413 This work was supported by the innovation team of Scientific Research Platform in Anhui 414 Province, the authors thank current and past members of Cai lab.

\section{REFERENCES}

416 417

418

419

420

421
Abraham RT. 2001. Cell cycle checkpoint signaling through the ATM and ATR kinases. Genes Dev 15:2177-2196. 10.1101/gad.914401

Anders S, and Huber W. 2010. Differential expression analysis for sequence count data. Genome Biol 11:R106. 10.1186/gb-2010-11-10-r106

Bagatell R, and Cohn SL. 2016. Genetic discoveries and treatment advances in neuroblastoma. Curr Opin Pediatr 28:19-25. 10.1097/MOP.0000000000000296 
422

423

424

425

426

427

428

429

430

431

432

433

434

435

436

437

438

439

440

441

442

443

444

445

446

447

448

449

450

451

452

453

454

455

456

457

458

459

460

461

462

Binkhathlan Z, and Lavasanifar A. 2013. P-glycoprotein inhibition as a therapeutic approach for overcoming multidrug resistance in cancer: current status and future perspectives. Curr Cancer Drug Targets 13:326-346.

Bleau AM, Hambardzumyan D, Ozawa T, Fomchenko EI, Huse JT, Brennan CW, and Holland EC. 2009. PTEN/PI3K/Akt pathway regulates the side population phenotype and ABCG2 activity in glioma tumor stem-like cells. Cell Stem Cell 4:226-235. 10.1016/j.stem.2009.01.007

Cai Y, Li J, Yang S, Ping L, Xuan Z, and Liu H. 2012. CIBZ, a Novel BTB Domain-Containing Protein, Is Involved in Mouse Spinal Cord Injury via Mitochondrial Pathway Independent of p53 Gene. PLoS One 7:e33156. 10.1371/journal.pone.0033156

Cai Y, Li J, Zhang Z, Chen J, Zhu Y, Li R, Chen J, Gao L, Liu R, and Teng Y. 2017. Zbtb38 is a novel target for spinal cord injury. Oncotarget 8:45356-45366. 10.18632/oncotarget.17487

Carnero A, Blanco-Aparicio C, Renner O, Link W, and Leal JF. 2008. The PTEN/PI3K/AKT signaling pathway in cancer, therapeutic implications. Curr Cancer Drug Targets 8:187-198.

Castel V, Segura V, and Berlanga P. 2013. Emerging drugs for neuroblastoma. Expert Opin Emerg Drugs 18:155-171. 10.1517/14728214.2013.796927

Chang Z, Li G, Liu J, Zhang Y, Ashby C, Liu D, Cramer CL, and Huang X. 2015. Bridger: a new framework for de novo transcriptome assembly using RNA-seq data. Genome Biol 16:30. 10.1186/s13059-015-0596-2

Dragoi AM, and Agaisse H. 2015. The class II phosphatidylinositol 3-phosphate kinase PIK3C2A promotes Shigella flexneri dissemination through formation of vacuole-like protrusions. Infect Immun 83:1695-1704. 10.1128/IAI.03138-14

Ewing B, and Green P. 1998. Base-calling of automated sequencer traces using phred. II. Error probabilities. Genome Res 8:186-194.

Ewing B, Hillier L, Wendl MC, and Green P. 1998. Base-calling of automated sequencer traces using phred. I. Accuracy assessment. Genome Res 8:175-185.

Florea L, Song L, and Salzberg SL. 2013. Thousands of exon skipping events differentiate among splicing patterns in sixteen human tissues. F1000Res 2:188. 10.12688/f1000research.2-188.v2

Ganley IG, Lam du H, Wang J, Ding X, Chen S, and Jiang X. 2009. ULK1.ATG13.FIP200 complex mediates mTOR signaling and is essential for autophagy. J Biol Chem 284:12297-12305. 10.1074/jbc.M900573200

Gotz S, Garcia-Gomez JM, Terol J, Williams TD, Nagaraj SH, Nueda MJ, Robles M, Talon M, Dopazo J, and Conesa A. 2008. High-throughput functional annotation and data mining with the Blast2GO suite. Nucleic Acids Res 36:3420-3435. 10.1093/nar/gkn176

Han W, and Wang HM. 2015. Refractory diarrhea: A paraneoplastic syndrome of neuroblastoma. World J Gastroenterol 21:7929-7932. 10.3748/wjg.v21.i25.7929

Jardin F, Ruminy P, Bastard C, and Tilly H. 2007. The BCL6 proto-oncogene: a leading role during germinal center development and lymphomagenesis. Pathol Biol (Paris) 55:73-83. 10.1016/j.patbio.2006.04.001

Jin M, Park SJ, Kim SW, Kim HR, Hyun JW, and Lee JH. 2017. PIG3 Regulates p53 Stability by Suppressing Its MDM2-Mediated Ubiquitination. Biomol Ther (Seoul) 25:396-403. 10.4062/biomolther.2017.086 
463 Kakarougkas A, Ismail A, Chambers AL, Riballo E, Herbert AD, Kunzel J, Lobrich M, Jeggo PA, and

464

465

466

467

468

469

470

471

472

473

474

475

476

477

478

479

480

481

482

483

484

485

486

487

488

489

490

491

492

493

494

495

496

497

498

499

500

501

502

503 Downs JA. 2014. Requirement for PBAF in transcriptional repression and repair at DNA breaks in actively transcribed regions of chromatin. Mol Cell 55:723-732. 10.1016/j.molcel.2014.06.028

Kanehisa M, Araki M, Goto S, Hattori M, Hirakawa M, Itoh M, Katayama T, Kawashima S, Okuda S, Tokimatsu T, and Yamanishi Y. 2008. KEGG for linking genomes to life and the environment. Nucleic Acids Res 36:D480-484. 10.1093/nar/gkm882

Kim D, Pertea G, Trapnell C, Pimentel H, Kelley R, and Salzberg SL. 2013. TopHat2: accurate alignment of transcriptomes in the presence of insertions, deletions and gene fusions. Genome Biol 14:R36. 10.1186/gb-2013-14-4-r36

Kim E, and Shohet J. 2009. Targeted molecular therapy for neuroblastoma: the ARF/MDM2/p53 axis. $J$ Natl Cancer Inst 101:1527-1529. 10.1093/jnci/djp376

Langmead B, Trapnell C, Pop M, and Salzberg SL. 2009. Ultrafast and memory-efficient alignment of short DNA sequences to the human genome. Genome Biol 10:R25. 10.1186/gb-2009-10-3-r25

Lee JH, Kang Y, Khare V, Jin ZY, Kang MY, Yoon Y, Hyun JW, Chung MH, Cho SI, Jun JY, Chang IY, and You HJ. 2010. The p53-inducible gene 3 (PIG3) contributes to early cellular response to DNA damage. Oncogene 29:1431-1450. 10.1038/onc.2009.438

Lee SU, and Maeda T. 2012. POK/ZBTB proteins: an emerging family of proteins that regulate lymphoid development and function. Immunol Rev 247:107-119. 10.1111/j.1600-065X.2012.01116.x

Li B, Fillmore N, Bai Y, Collins M, Thomson JA, Stewart R, and Dewey CN. 2014. Evaluation of de novo transcriptome assemblies from RNA-Seq data. Genome Biol 15:553. 10.1186/s13059-0140553-5

Lord CJ, and Ashworth A. 2012. The DNA damage response and cancer therapy. Nature 481:287-294. 10.1038 /nature 10760

Maeda T, Merghoub T, Hobbs RM, Dong L, Maeda M, Zakrzewski J, van den Brink MR, Zelent A, Shigematsu H, Akashi K, Teruya-Feldstein J, Cattoretti G, and Pandolfi PP. 2007. Regulation of B versus T lymphoid lineage fate decision by the proto-oncogene LRF. Science 316:860-866. 10.1126/science. 1140881

Mao X, Cai T, Olyarchuk JG, and Wei L. 2005. Automated genome annotation and pathway identification using the KEGG Orthology (KO) as a controlled vocabulary. Bioinformatics 21:3787-3793. 10.1093/bioinformatics/bti430

Matsuda E, Yu O, and Kawaichi M. 2008. CIBZ,a BTB-containing Zinc Finger Protein,Plays a Role in Apoptosis and tumorigenesis. bit life sciences' 1st annual world cancer congess-2008.

Matsuoka S, Ballif BA, Smogorzewska A, McDonald ER, 3rd, Hurov KE, Luo J, Bakalarski CE, Zhao Z, Solimini N, Lerenthal Y, Shiloh Y, Gygi SP, and Elledge SJ. 2007. ATM and ATR substrate analysis reveals extensive protein networks responsive to DNA damage. Science 316:1160-1166. 10.1126/science. 1140321

McGettigan PA. 2013. Transcriptomics in the RNA-seq era. Curr Opin Chem Biol 17:4-11. 10.1016/j.cbpa.2012.12.008

Nishii T, Oikawa Y, Ishida Y, Kawaichi M, and Matsuda E. 2012. CtBP-interacting BTB zinc finger protein (CIBZ) promotes proliferation and G1/S transition in embryonic stem cells via Nanog. Journal of Biological Chemistry 287:12417. 10.1074/jbc.M111.333856 
504 Phan RT, and Dalla-Favera R. 2004. The BCL6 proto-oncogene suppresses p53 expression in germinal-

505

506

507

508

509

510

511

512

513

514

515

516

517

518

519

520

521

522

523

524

525

526

527

528

529

530

531

532

533

534

535

536

537

538

539

540

541

542

543

544 centre B cells. Nature 432:635-639. 10.1038/nature03147

Redden RA, Iyer R, Brodeur GM, and Doolin EJ. 2014. Rotary bioreactor culture can discern specific behavior phenotypes in Trk-null and Trk-expressing neuroblastoma cell lines. In Vitro Cell Dev Biol Anim 50:188-193. 10.1007/s11626-013-9716-z

Reimann E, Koks S, Ho XD, Maasalu K, and Martson A. 2014. Whole exome sequencing of a single osteosarcoma case--integrative analysis with whole transcriptome RNA-seq data. Hum Genomics 8:20. 10.1186/s40246-014-0020-0

Robinson MD, McCarthy DJ, and Smyth GK. 2010. edgeR: a Bioconductor package for differential expression analysis of digital gene expression data. Bioinformatics 26:139-140. 10.1093/bioinformatics/btp616

Sasai N, Matsuda E, Sarashina E, Ishida Y, and Kawaichi M. 2005. Identification of a novel BTB-zinc finger transcriptional repressor, CIBZ, that interacts with CtBP corepressor. Genes to Cells 10:871-885. 10.1111/j.1365-2443.2005.00885.x

Sawai CM, Freund J, Oh P, Ndiaye-Lobry D, Bretz JC, Strikoudis A, Genesca L, Trimarchi T, Kelliher MA, Clark M, Soulier J, Chen-Kiang S, and Aifantis I. 2012. Therapeutic targeting of the cyclin D3:CDK4/6 complex in T cell leukemia. Cancer Cell 22:452-465. 10.1016/j.ccr.2012.09.016

Saxton, R. A., \& Sabatini, D. M. 2017. Mtor signaling in growth, metabolism, and disease. Cell 168(6), 960-976. 10.1016/j.cell.2017.02.004.

Schulte JH, Schulte S, Heukamp LC, Astrahantseff K, Stephan H, Fischer M, Schramm A, and Eggert A. 2013. Targeted Therapy for Neuroblastoma: ALK Inhibitors. Klin Padiatr 225:303-308. $10.1055 / \mathrm{s}-0033-1357132$

Shangary S, and Wang S. 2009. Small-molecule inhibitors of the MDM2-p53 protein-protein interaction to reactivate p53 function: a novel approach for cancer therapy. Annu Rev Pharmacol Toxicol 49:223-241. 10.1146/annurev.pharmtox.48.113006.094723

Shi Y, Gao X, Hu Q, Li X, Xu J, Lu S, Liu Y, Xu C, Jiang D, Lin J, Xue A, Tan Y, Shen K, and Hou Y. 2016. PIK3C2A is a gene-specific target of microRNA-518a-5p in imatinib mesylate-resistant gastrointestinal stromal tumor. Lab Invest 96:652-660. 10.1038/labinvest.2015.157

Stogios PJ, Downs GS, Jauhal JJ, Nandra SK, and Prive GG. 2005. Sequence and structural analysis of BTB domain proteins. Genome Biol 6:R82. 10.1186/gb-2005-6-10-r82

The I, Ruijtenberg S, Bouchet BP, Cristobal A, Prinsen MB, van Mourik T, Koreth J, Xu H, Heck AJ, Akhmanova A, Cuppen E, Boxem M, Munoz J, and van den Heuvel S. 2015. Rb and FZR1/Cdh1 determine CDK4/6-cyclin D requirement in C. elegans and human cancer cells. Nat Commun 6:5906. 10.1038/ncomms6906

VanArsdale T, Boshoff C, Arndt KT, and Abraham RT. 2015. Molecular Pathways: Targeting the Cyclin D-CDK4/6 Axis for Cancer Treatment. Clin Cancer Res 21:2905-2910. 10.1158/10780432.CCR-14-0816

Wang D, Olman MA, Stewart J, Jr., Tipps R, Huang P, Sanders PW, Toline E, Prayson RA, Lee J, Weil RJ, Palmer CA, Gillespie GY, Liu WM, Pieper RO, Guan JL, and Gladson CL. 2011. Downregulation of FIP200 induces apoptosis of glioblastoma cells and microvascular endothelial cells by enhancing Pyk2 activity. PLoS One 6:e19629. 10.1371/journal.pone.0019629 
545 Wang XW, Zhan Q, Coursen JD, Khan MA, Kontny HU, Yu L, Hollander MC, O'Connor PM, Fornace

546

547

548

549

550

551

552

553

554

555

556

557

558

559

560

561

562

563

564

565

566

567

568
AJ, Jr., and Harris CC. 1999. GADD45 induction of a G2/M cell cycle checkpoint. Proc Natl Acad Sci U S A 96:3706-3711.

Wang Z, Gerstein M, and Snyder M. 2009. RNA-Seq: a revolutionary tool for transcriptomics. Nat Rev Genet 10:57-63. 10.1038/nrg2484

Wei H, Gan B, Wu X, and Guan JL. 2009. Inactivation of FIP200 leads to inflammatory skin disorder, but not tumorigenesis, in conditional knock-out mouse models. J Biol Chem 284:6004-6013. 10.1074/jbc.M806375200

Wong BR, Besser D, Kim N, Arron JR, Vologodskaia M, Hanafusa H, and Choi Y. 1999. TRANCE, a TNF family member, activates Akt/PKB through a signaling complex involving TRAF6 and cSrc. Mol Cell 4:1041-1049.

Yang T, Massa SM, Tran KC, Simmons DA, Rajadas J, Zeng AY, Jang T, Carsanaro S, and Longo FM. 2016. A small molecule TrkB/TrkC neurotrophin receptor co-activator with distinctive effects on neuronal survival and process outgrowth. Neuropharmacology 110:343-361.

10.1016/j.neuropharm.2016.06.015

Young MD, Wakefield MJ, Smyth GK, and Oshlack A. 2010. Gene ontology analysis for RNA-seq: accounting for selection bias. Genome Biol 11:R14. 10.1186/gb-2010-11-2-r14

Zhang Z, Chen J, Chen F, Yu D, Li R, Lv C, Wang H, Li H, Li J, and Cai Y. 2017. Tauroursodeoxycholic acid alleviates secondary injury in the spinal cord via up-regulation of CIBZ gene. Cell Stress Chaperones. 10.1007/s12192-017-0862-1

Zhao QY, Wang Y, Kong YM, Luo D, Li X, and Hao P. 2011. Optimizing de novo transcriptome assembly from short-read RNA-Seq data: a comparative study. BMC Bioinformatics 12 Suppl 14:S2. 10.1186/1471-2105-12-S14-S2 


\section{Figure 1}

Expression analysis of ZBTB38 gene in different tumors based on TCGA Database.

(A) The expression changes of ZBTB38 gene are closely related to the occurrence of 20 kinds of cancers; (B-D) ZBTB38 expression profiles based on top 4 cancer stages. ${ }^{*} p<0.05$; ${ }^{* *} p<$ 0.01 . 
A

\section{Cancer Distribution}

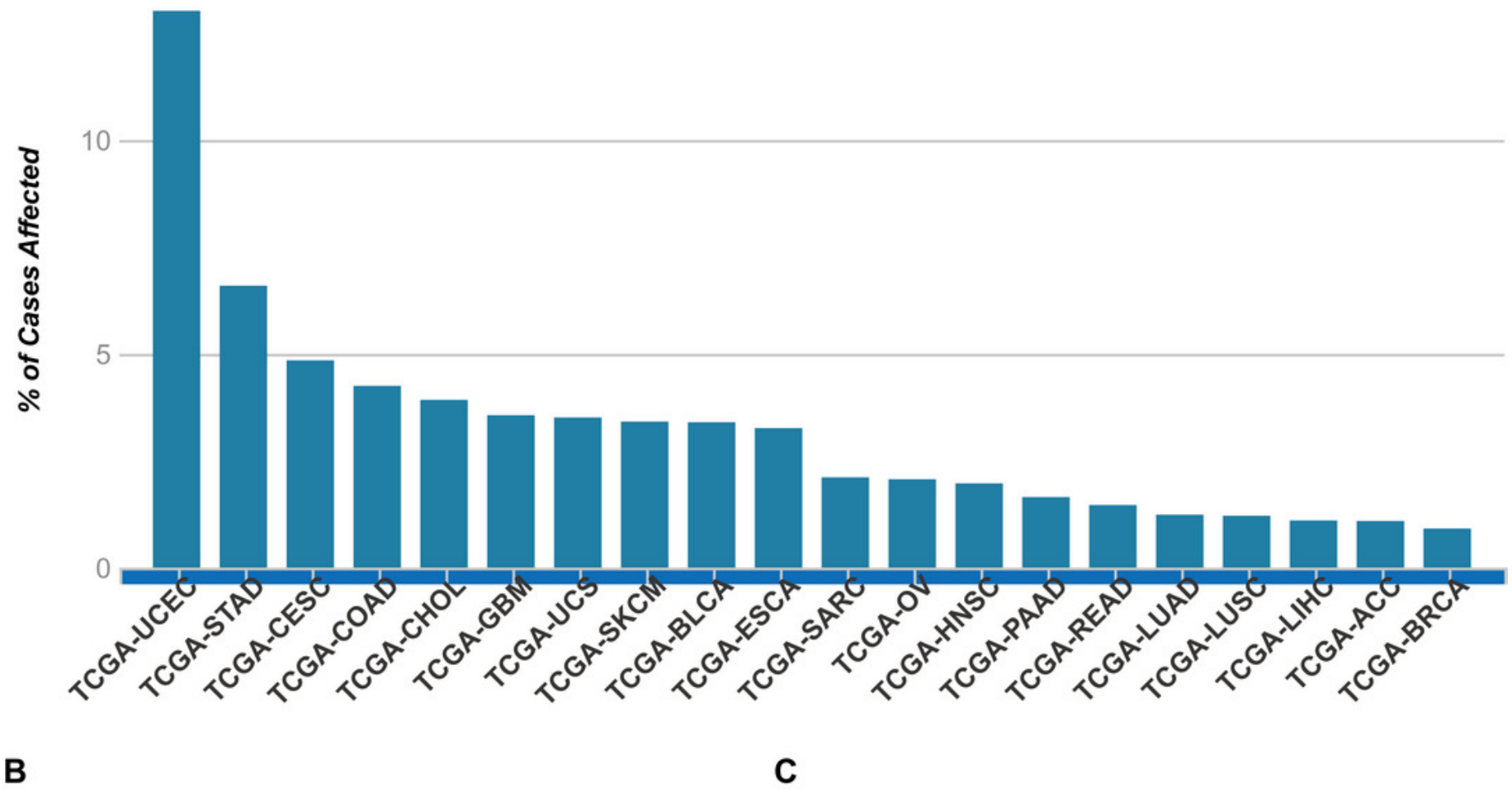

Expression of ZBTB38 in UCEC based on major cancer stages

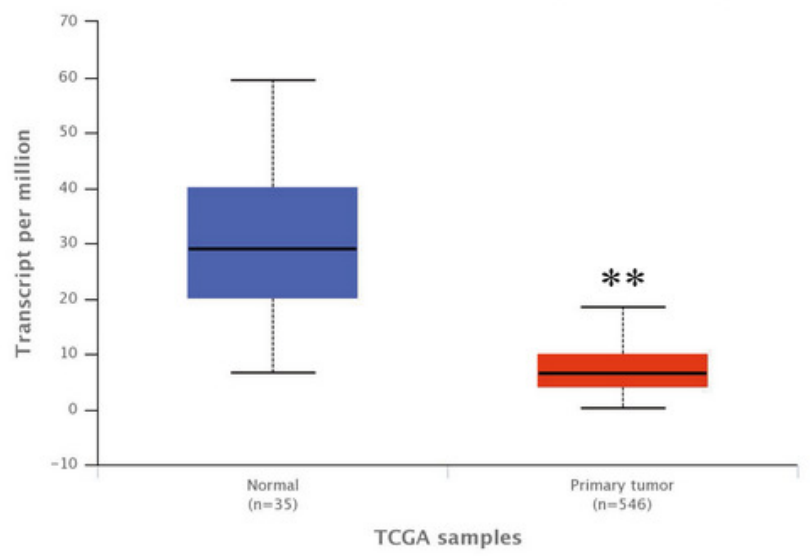

D
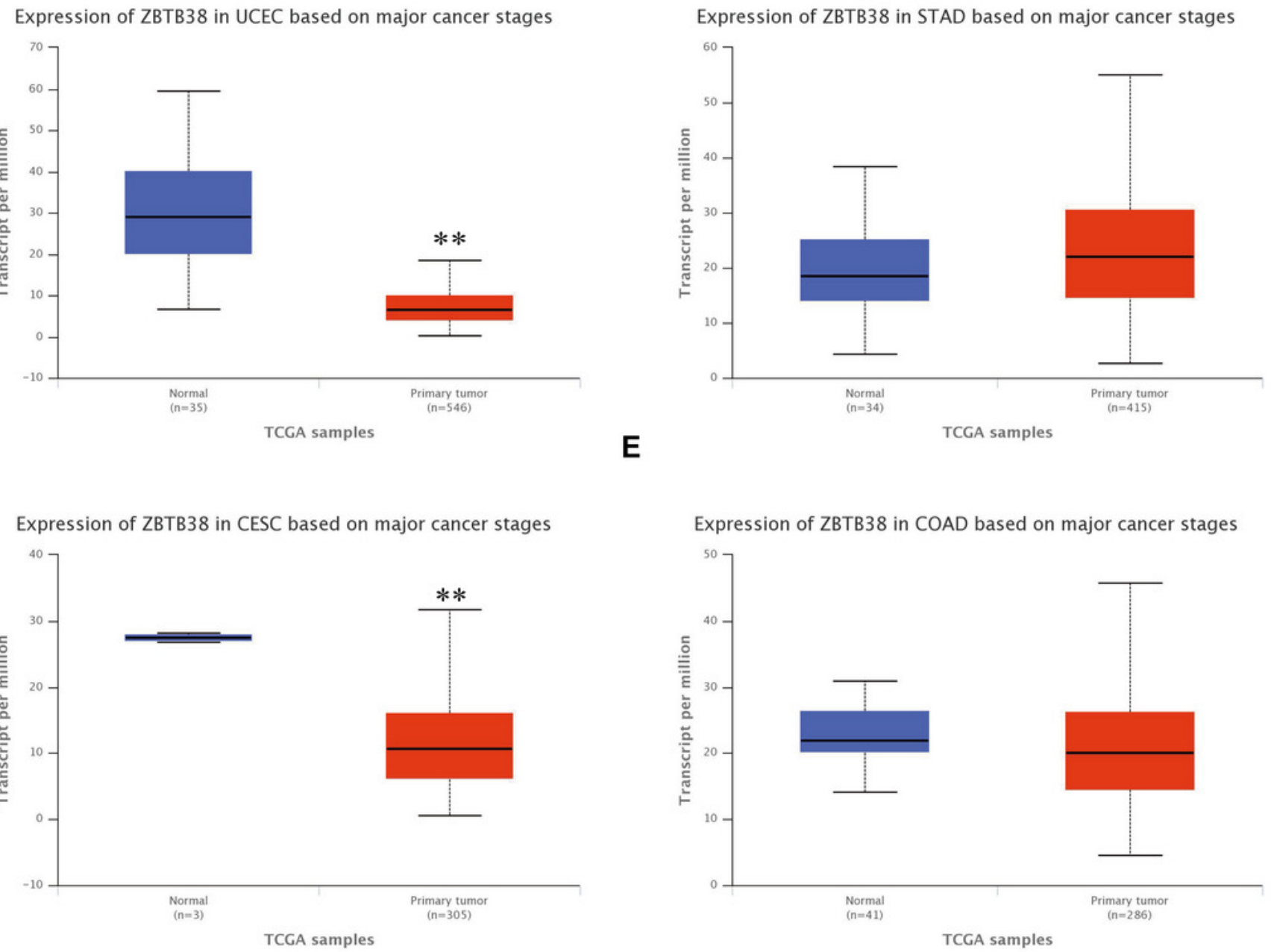
Figure 2

Effect of ZBTB38 expression level on LGG patient survival.

Red and blue lines indicated high and low expression groups, respectively. $P=0.02<0.05$ was considered to be statistically significant.

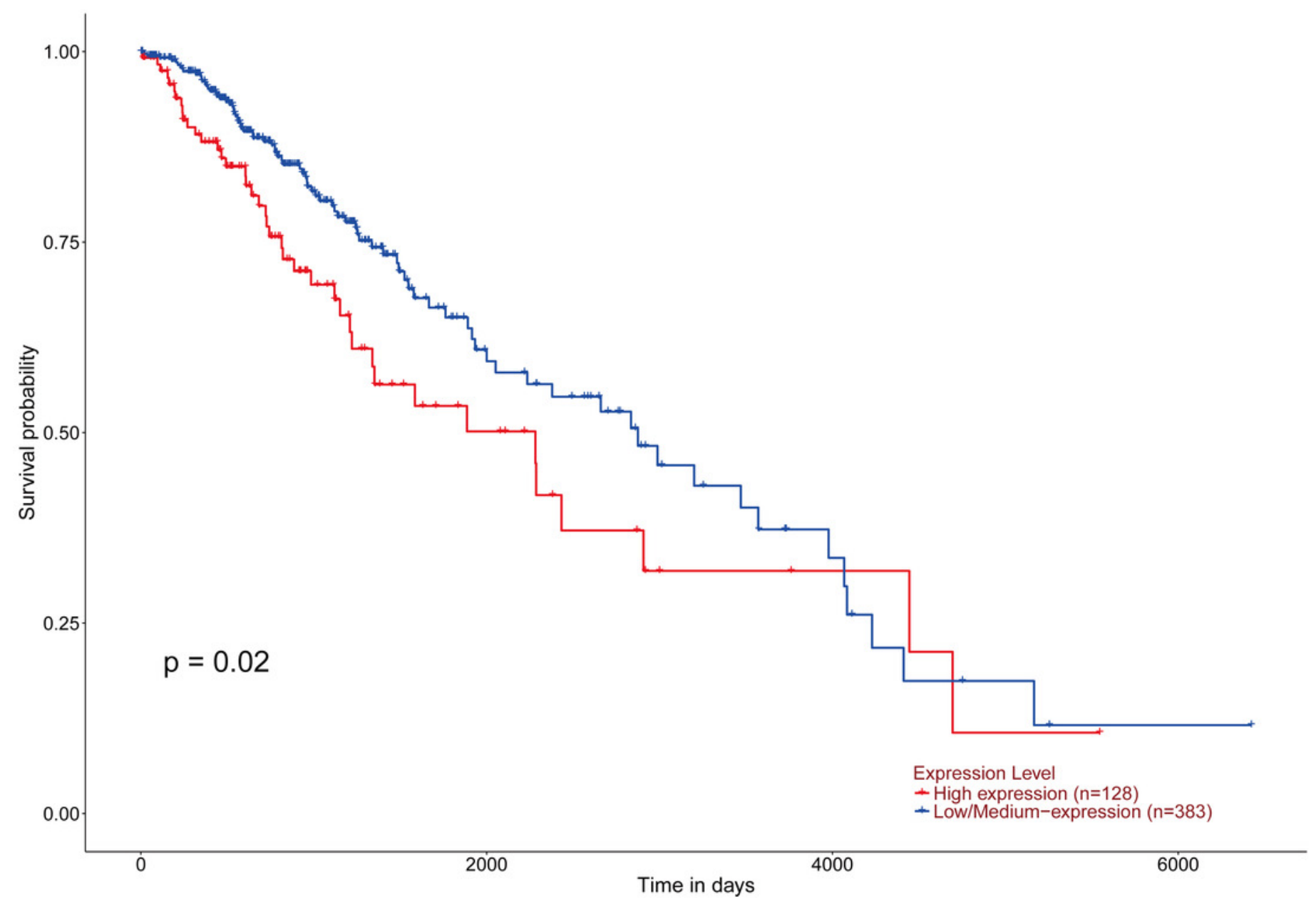




\section{Figure 3}

Proliferation and viability of ZBTB38 knockdown SH-SY5Y cells.

(A, B) SH-SY5Y cells were divided into two groups underwent control group and siRNA, respectively. cell lysates were collected for Western blot analysis on the $72 \mathrm{~h}$ after transfecting different sequences of ZBTB38 siRNAs (siRNA1, siRNA2 and siRNA3 indicate three different siRNA primers). Representative images of these assays are shown in (A) and quantitative data are shown in (B); $\beta$-actin was used as an internal control. SH-SY5Y cell proliferation (C) and viability (D) in different groups. ${ }^{*} p<0.05$. Data are presented as means \pm SEM from at least 3 independent experiments. Control, SH-SY5Y cells treated with liposome alone; ZBTB38\%", SH-SY5Y cells transfected with ZBTB38 siRNA.

A

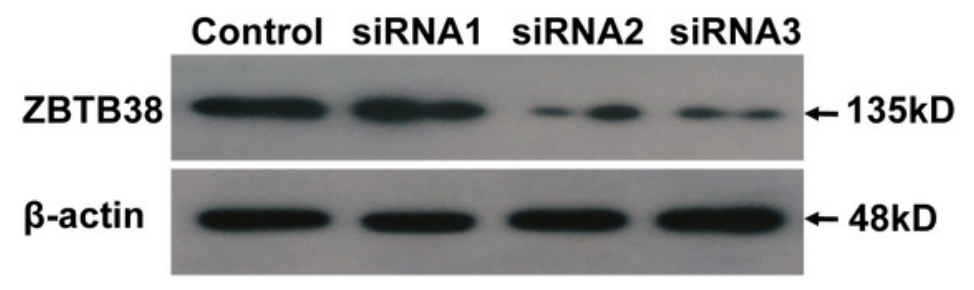

C

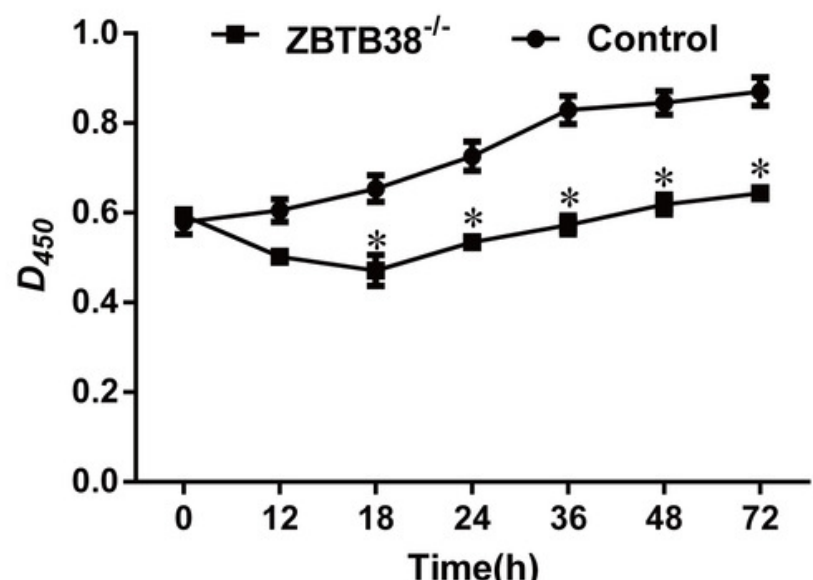

B

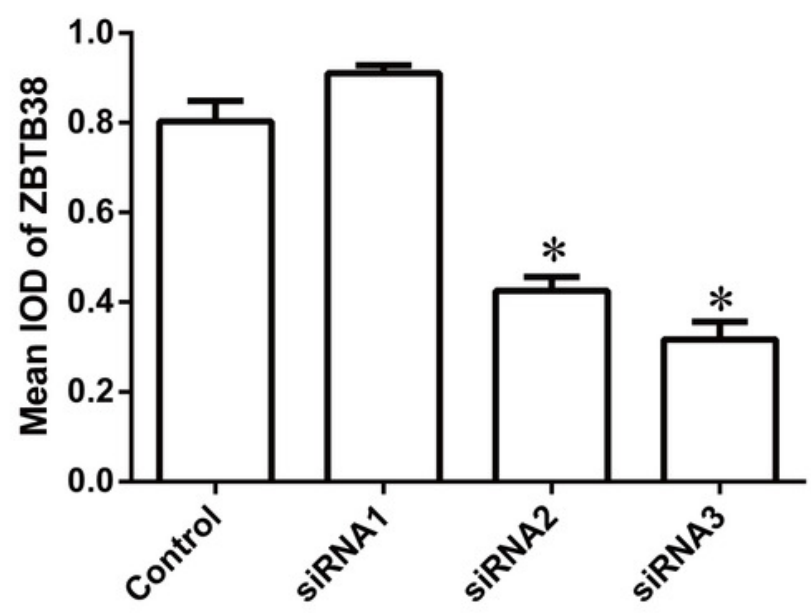

D

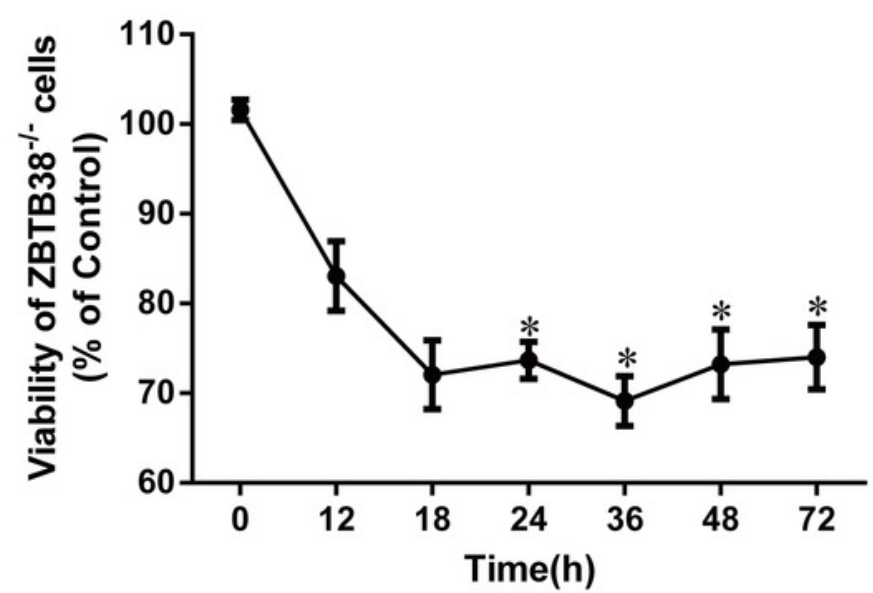


Figure 4

Loss of ZBTB38 induces apoptosis in SH-SY5Y cells.

(A) Apoptosis of SH-SY5Y cells in the presence or absence of ZBTB38 siRNA was determined

by The Cell Death Detection Elisa Kit. (B) RNA from ZBTB38 knockdown SH-SY5Y cells

(siZBTB38) and control cells (siNC) was collected for QRT-PCR analysis to determine the expression levels of pro-apoptotic genes. ${ }^{*} p<0.05 ; * * p<0.01$.

A

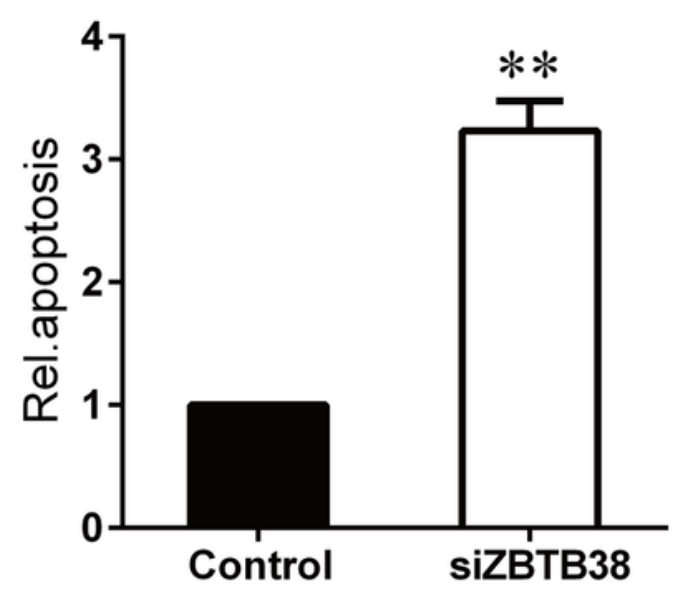

B

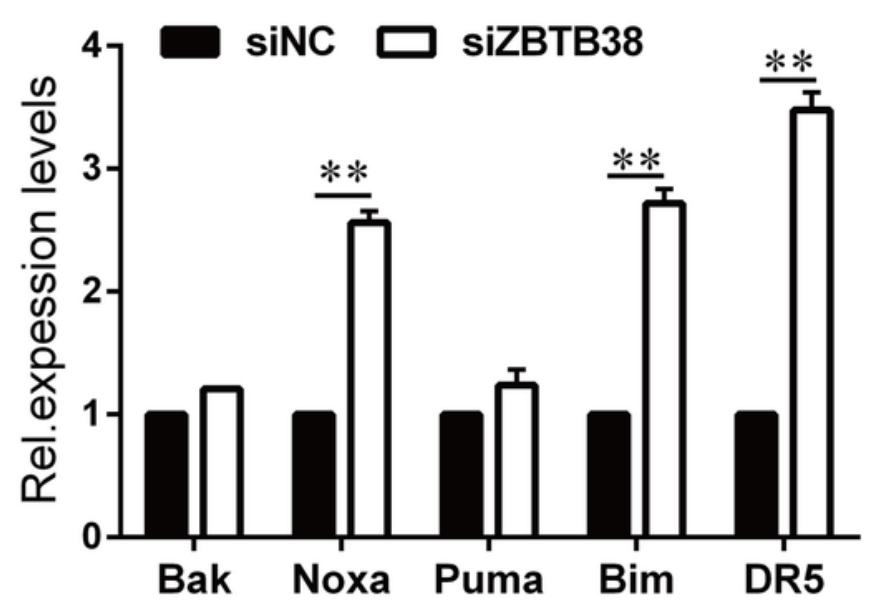


Figure 5

Gene function classification of all annotated unigenes by Gene Ontology.

The vertical axis represents the number of unigenes, and horizontal axis gives the specific GO sub-categories.

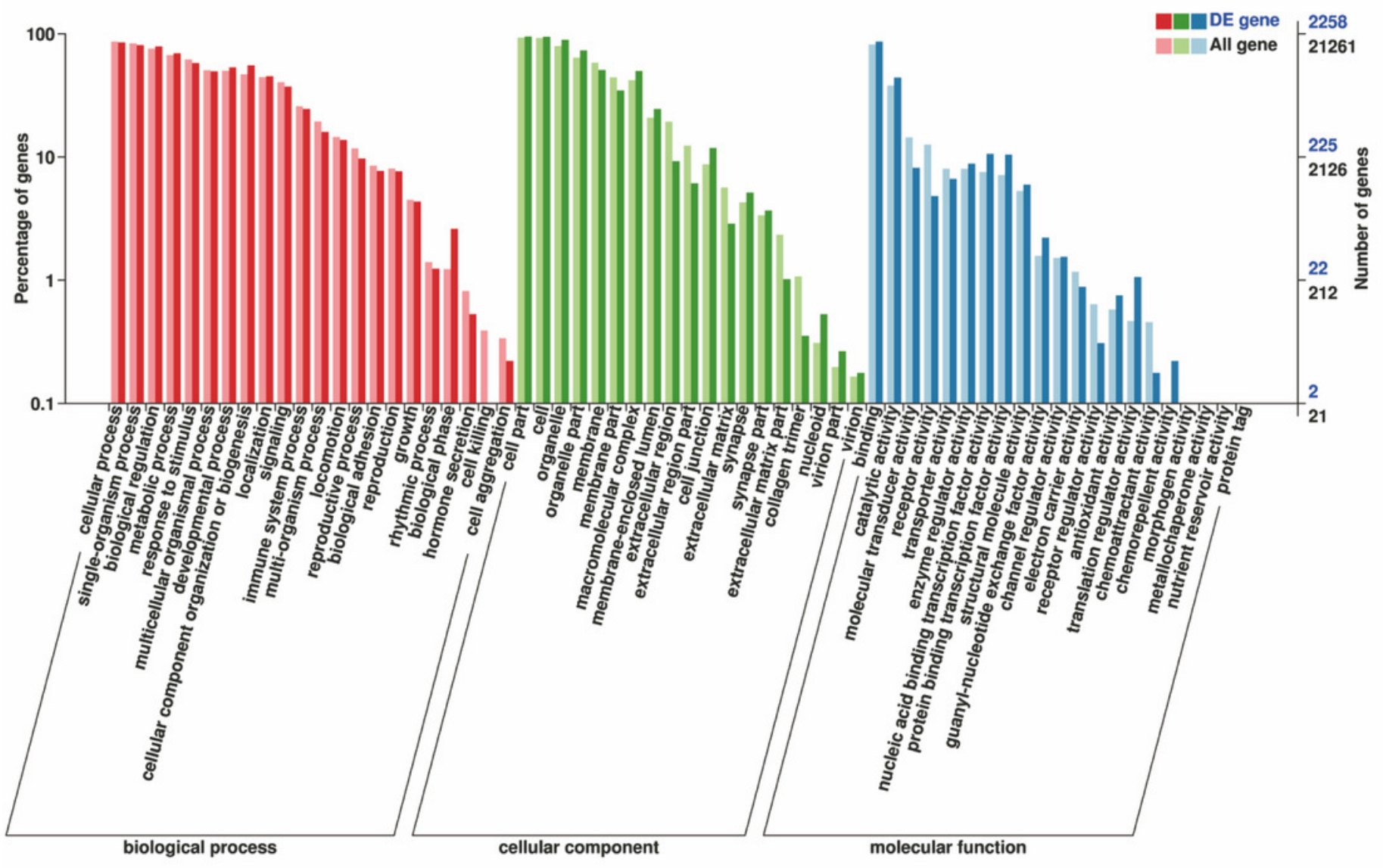




\section{Figure 6}

\section{COG function classification of consensus sequence.}

The vertical axis represents the frequency of unigenes classified into the specific categories, and horizontal axis gives the COG function classification.

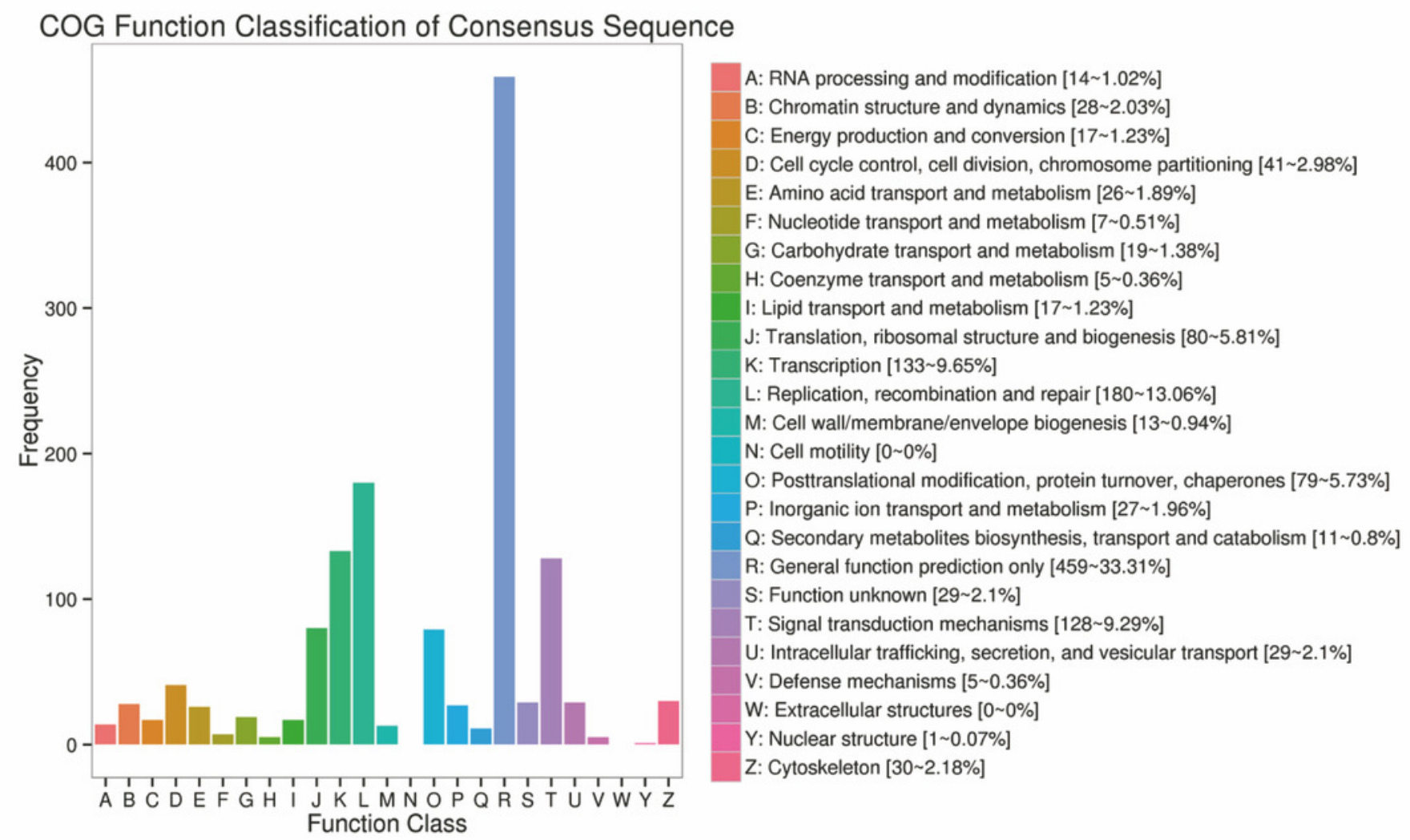


Figure 7

DEG KEGG classification.

The vertical axis lists the various metabolic pathways, and horizontal axis gives the number of annotated genes in the pathways.

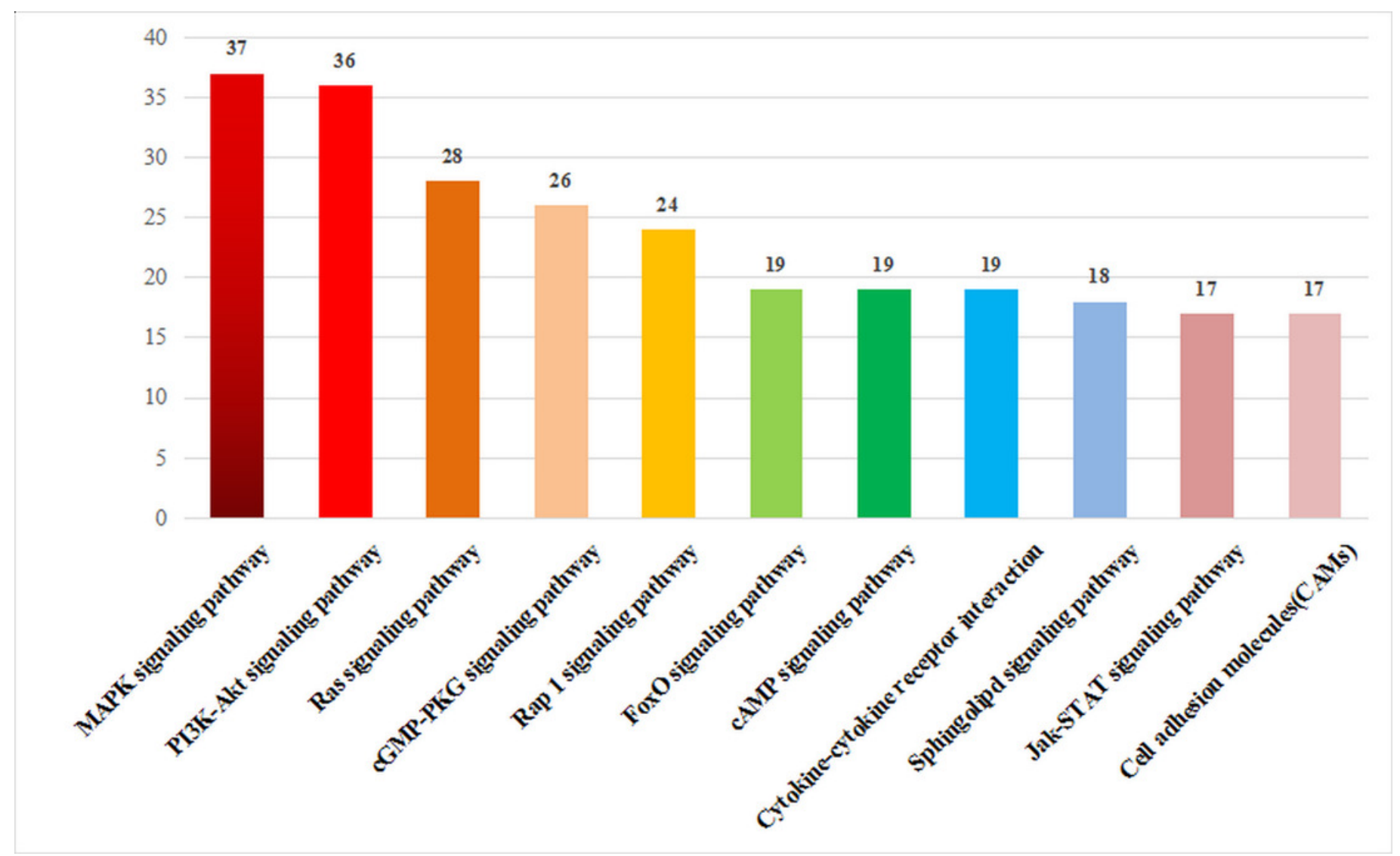


Figure 8

Differential expression analysis of candidate genes between ZBTB38 ${ }^{-1}$ and ZBTB38 SHSY5Y cells.

(A) The result of qRT-PCR. (B)The result of RNA-seq.

A

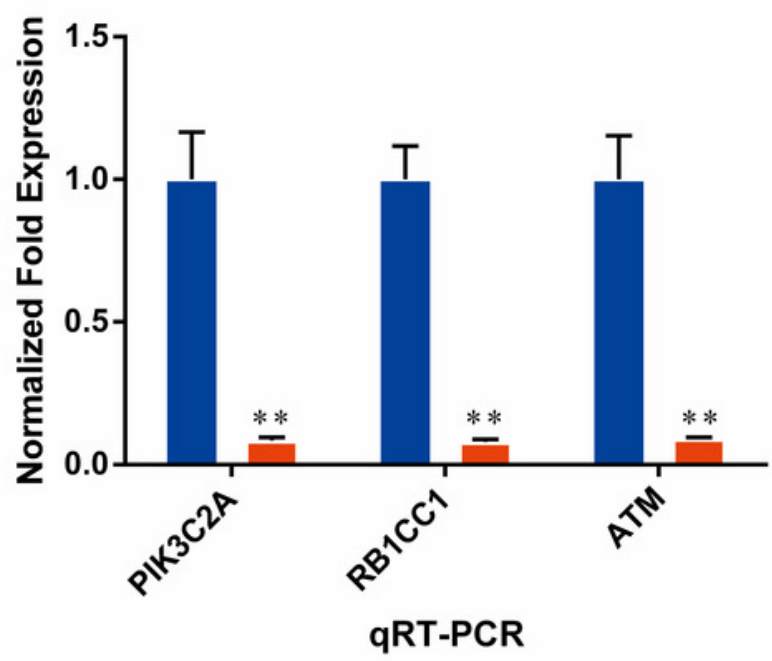

B

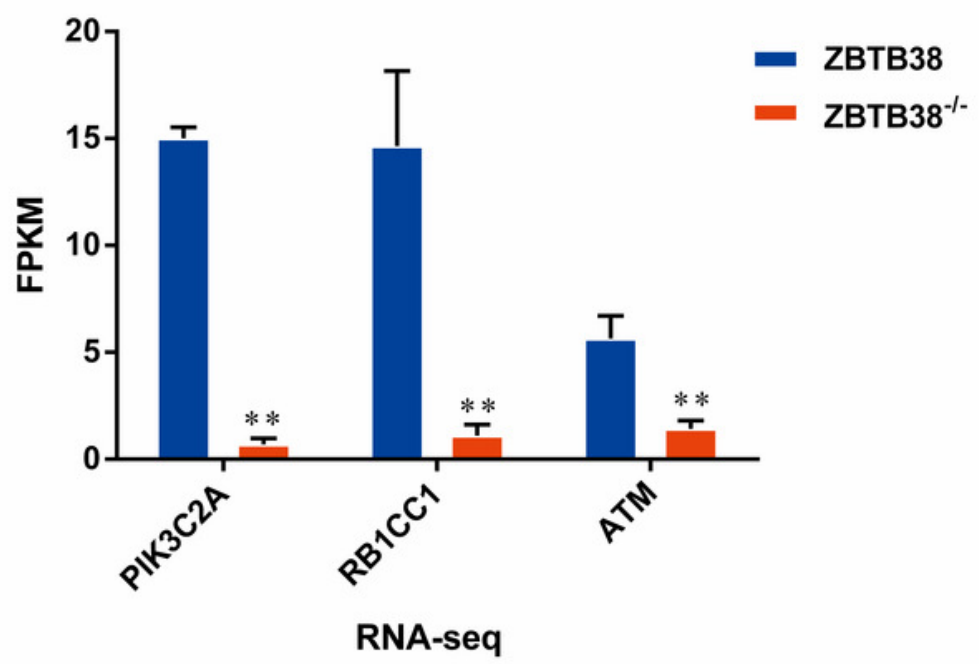


Figure 9

Knockdown of ZBTB38 triggers inhibition of autophagy in SH-SY5Y cells.

(A) SH-SY5Y cells were transfected with scramble siRNA (siNC) or siRNA against ZBTB38

(siZBTB38-2 and siZBTB38-3) for 72 hours and cell lysates were collected for Western blot analysis. (B) Quantification of protein expression was performed by densitometric analysis. ${ }^{*} p<0.05 ; * * p 0.01$.

A

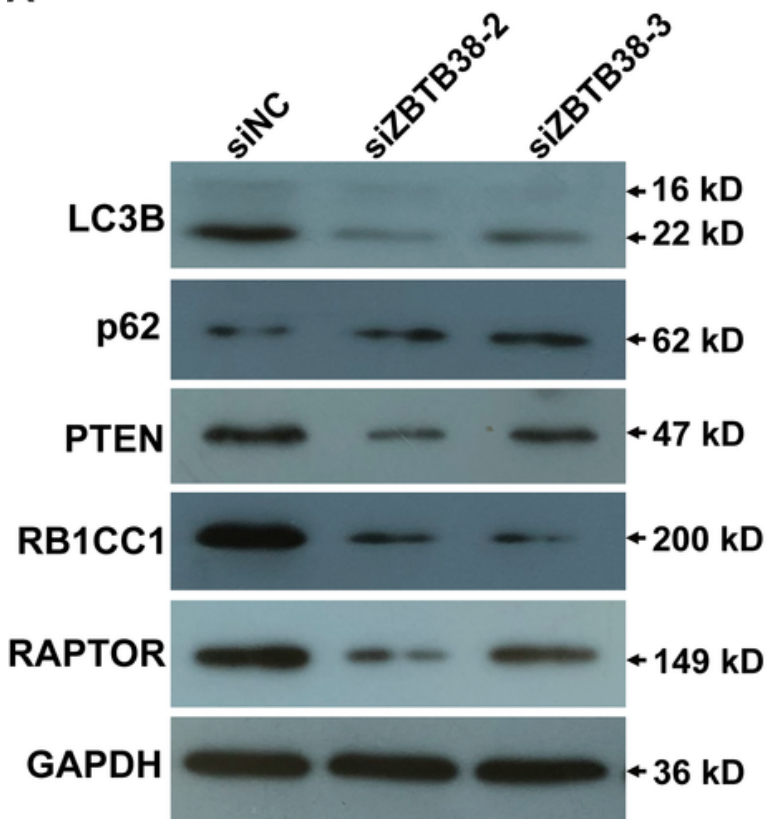

B

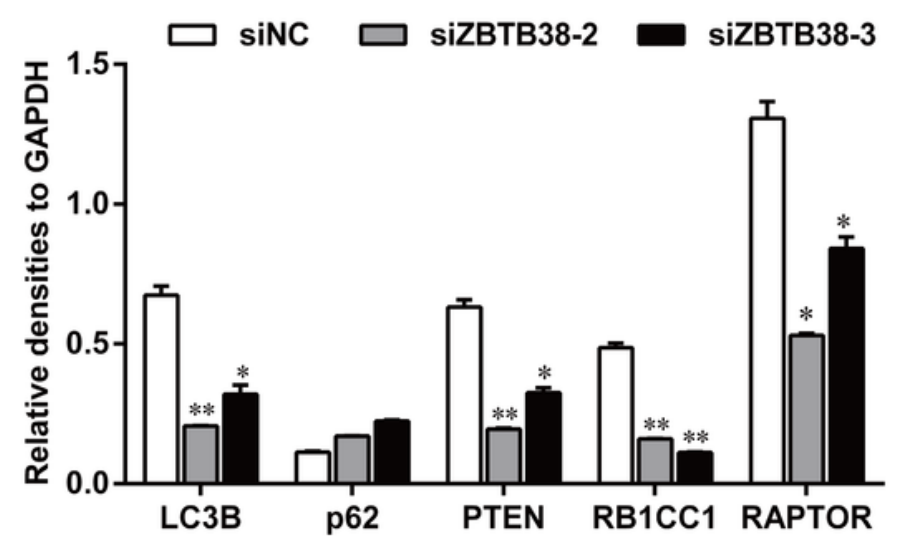




\section{Figure 10}

KEGG pathway annotation map of differentially expressed genes in p53 signaling pathway.

Relative to the control group, the red labeled protein was associated with the up-regulated gene and the white labeled protein was associated with the down-regulated gene.

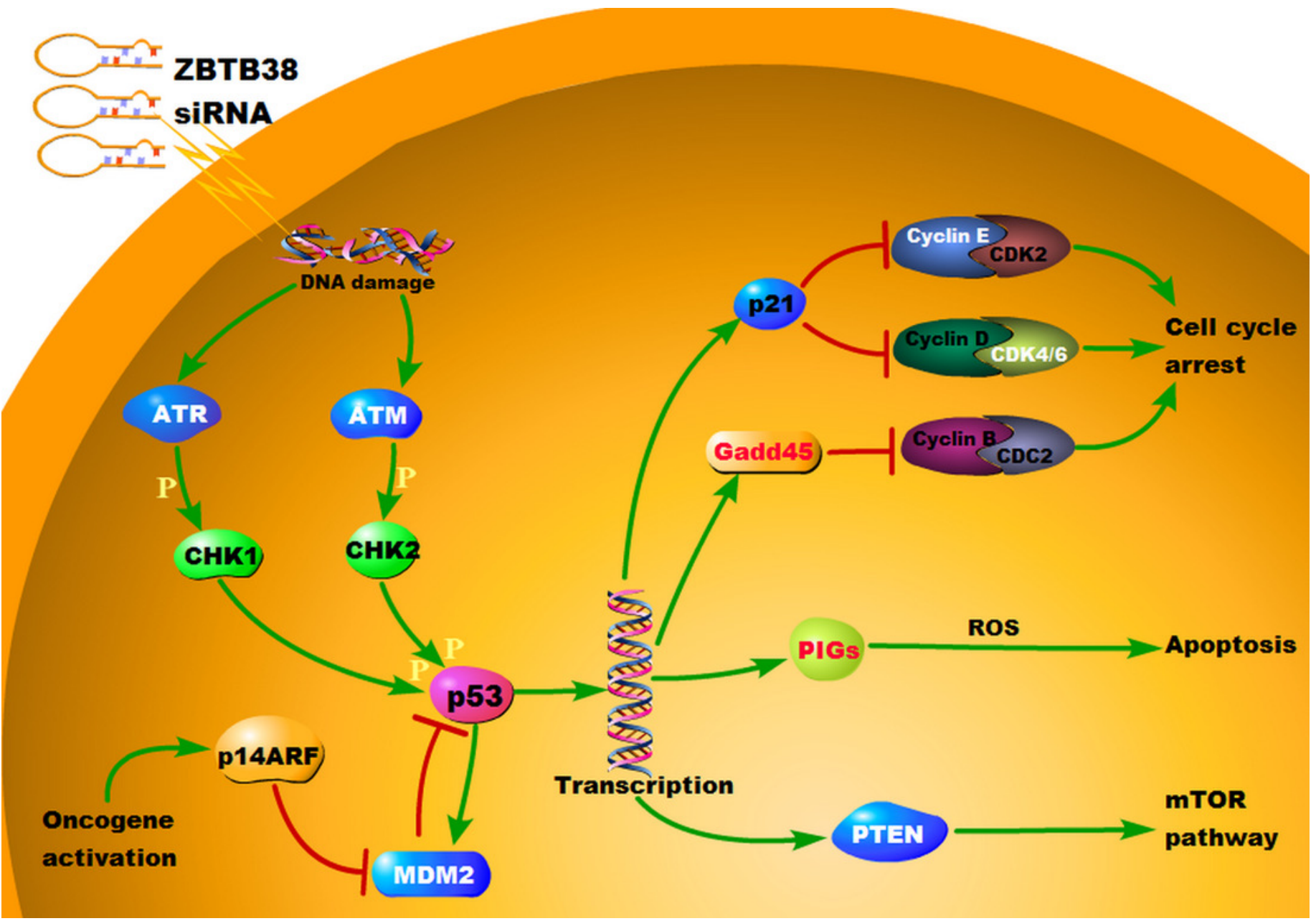




\section{Table $\mathbf{1}$ (on next page)}

Summary of Sequence comparisons among sample sequencing data and selected reference genomes.

T01, T02, and T07 indicate the ZBTB38- SH-SY5Y cells. T04, T05, and T06 indicate the control groups. Total Reads: Number of Clean Reads, single-ended; Mapped Reads: Number of Reads aligned to the reference genome and percentage in Clean Reads; Uniq Mapped Reads: Match The number of Reads to the unique position of the reference genome and the percentage of the Clean Reads; Multiple Map Reads: The number of Reads aligned to multiple locations in the reference genome and the percentage of Clean Reads.GC content: The Clean Data GC content; $\geq$ Q30\% : The percentage of bases with a Clean Data quality value $\geq 30$. 


\begin{tabular}{|c|c|c|c|c|cc}
\hline Samples-ID & Total Reads & Mapped Reads & Uniq Mapped Reads & Multiple Map Reads & GC Content & $\% \geq$ Q30 \\
\hline T01 & $41,320,306$ & $32,993,483(79.85 \%)$ & $29,171,906(70.60 \%)$ & $3,821,577(9.25 \%)$ & $56.24 \%$ & $89.44 \%$ \\
\hline T02 & $53,706,092$ & $42,655,511(79.42 \%)$ & $37,610,672(70.03 \%)$ & $5,044,839(9.39 \%)$ & $55.36 \%$ & $89.45 \%$ \\
\hline T04 & $54,889,928$ & $44,964,104(81.92 \%)$ & $41,561,590(75.72 \%)$ & $3,402,514(6.20 \%)$ & $52.17 \%$ & $90.04 \%$ \\
\hline T05 & $50,160,236$ & $40,559,313(80.86 \%)$ & $37,552,835(74.87 \%)$ & $3,006,478(5.99 \%)$ & $52.16 \%$ & $90.25 \%$ \\
\hline T06 & $62,721,676$ & $50,526,963(80.56 \%)$ & $47,347,475(75.49 \%)$ & $3,179,488(5.07 \%)$ & $51.94 \%$ & $90.05 \%$ \\
\hline T07 & $54,693,534$ & $43,544,178(79.61 \%)$ & $38,543,679(70.47 \%)$ & $5,000,499(9.14 \%)$ & $55.22 \%$ & $89.30 \%$ \\
\hline
\end{tabular}

2 
Table 2 (on next page)

Summary of the function annotation results for ZBTB38-1 unigenes in public protein databases. 


\begin{tabular}{lllllllll}
\hline DEG Set & Total & COG & GO & KEGG & KOG & NR & Swiss-Prot & eggNOG \\
\hline $\begin{array}{l}\text { T04_T05_T06 vs } \\
\text { T01_T02_T07 }\end{array}$ & 2,417 & 999 & 2,258 & 1,512 & 1,733 & 2,337 & 2,377 & 2,405 \\
\hline
\end{tabular}

1 


\section{Table 3(on next page)}

TopGO enrichment results of differential expression genes.

Term: GO function; Annotated: The number of genes annotated to this function for all genes;

Significant: The number of genes annotated to this function in the DEG; Expected: The expected value of the number of DEGs for this function; KS: Statistical significance of enriched Term, the smaller the KS value, the more significant the enrichment. 


\begin{tabular}{|c|c|c|c|c|c|}
\hline GO:0048011 & Neurotrophin TRK receptor signaling pathway & 562 & 68 & 60.42 & $9.00 \mathrm{E}-14$ \\
\hline GO:0045893 & Positive regulation of transcription, DNA-templated & 2389 & 314 & 256.83 & $2.90 \mathrm{E}-13$ \\
\hline GO:0007268 & Synaptic transmission & 1693 & 160 & 182 & $6.90 \mathrm{E}-13$ \\
\hline GO:0044281 & Small molecule metabolic process & 5268 & 511 & 566.33 & $2.20 \mathrm{E}-11$ \\
\hline GO:0051656 & Establishment of organelle localization & 626 & 123 & 67.3 & $1.90 \mathrm{E}-10$ \\
\hline GO:0019219 & $\begin{array}{l}\text { Regulation of nucleobase-containing compound } \\
\text { metabolic process }\end{array}$ & 7440 & 1046 & 799.83 & $4.40 \mathrm{E}-10$ \\
\hline
\end{tabular}

